\title{
FRACTURAS Y REPRESENTACIÓN POLÍTICA EN EL MOVIMIENTO ESTUDIANTIL. CHILE 2011
}

\author{
OCTAVIO AVENDAÑO*
}

\begin{abstract}
RESUMEN
Este artículo analiza las diferencias ideológicas y políticas que surgieron entre los dirigentes del movimiento estudiantil universitario en la coyuntural del 2011. Tomando como referencia la teoría de los «clivajes», se plantea que durante ese año las propuestas y la representación del movimiento estudiantil se erigieron sobre una nueva fractura derivada de la crítica a la mercantilización de la Educación Superior. A medida que transcurren las movilizaciones, se fueron evidenciando otras diferencias entre los dirigentes de las principales organizaciones y federaciones estudiantiles, en torno a las negociaciones con el gobierno, la representación, la participación, la toma de decisiones y la proyección del movimiento.
\end{abstract}

PALABRAS CLAVE: MOVIMIENTO ESTUDIANTIL, FRACTURAS IDEOLÓGICAS, LIDERAZGOS ESTUDIANTILES

* Doctor en Ciencia Política, Università degli studi di Firenze, Italia. Académico del Departamento de Ciencia Política y Relaciones Internacionales de la Universidad Alberto Hurtado y del Departamento de Sociología de la Universidad de Chile.

E-Mail: oavendan@uahurtado.cl, oavendanop@u.uchile.cl.

Este artículo ha sido elaborado en el marco del proyecto Fondecyt Regular N¹140639, titulado: «Representación de intereses en Chile». 


\title{
FRATURAS E REPRESENTAÇÃO POLÍTICA NO MOVIMENTO ESTUDANTIL. CHILE 2011
}

\begin{abstract}
RESUMO
Este artigo analisa as diferenças ideológicas e políticas que surgiram entre os dirigentes do movimento estudantil universitário durante o ano 2011. Tomando como referência a teoria das clivagens, no artigo é apresentada a ideia de que durante esse ano os protestos e a representação do movimento estudantil foram erigidos sobre uma nova fratura derivada das críticas à mercantilização da Educação Superior. À medida que transcorriam as mobilizações, evidenciaram-se outras diferenças entre os dirigentes das principais organizações e federações estudantis, em torno às negociações com o governo, à representação, à participação, à tomada de decisões e à projeção do movimento.

\section{PALAVRAS CHAVE: MOVIMIENTO ESTUDIANTIL, FRACTURAS IDEOLÓGICAS,} LIDERANÇA ESTUDANTIL
\end{abstract}

\section{FRACTURES AND POLITICAL REPRESENTATION IN THE STUDENT MOVEMENT. CHILE 2011.}

\begin{abstract}
The following article analyses the ideological and political differences emerging among the leaders of the university student movement during 2011. On the basis of the theory of cleavages, it is suggested that students' proposals and their representation built on a new social fracture, the latter being an outcome of their criticism of mercantilization of higher education. Throughout demonstrations, other differences among the student leaders from the most important student organizations and federations were made evident, especially concerning issues such as negotiations with the government, representation, participation, decision-making process and the movement's future.
\end{abstract}

KEY WORDS: STUDENT MOVEMENT, IDEOLOGICAL FRACTURES, STUDENT LEADERSHIP 


\section{INTRODUCCIÓN}

LAS MOVILIZACIONES QUE SE registraron durante el año 2011 marcaron un punto de inflexión en el ciclo político inaugurado a partir de 1990. El movimiento estudiantil emprendió una serie de acciones que concitaron la adhesión de buena parte de la población. Además, fue capaz de involucrar a otros actores del ámbito educacional, junto con articular sus demandas e iniciativas con las de otros movimientos sociales que irrumpieron durante el 2011. El impacto de las acciones emprendidas por el movimiento de estudiantes universitarios fue tal que logró incidir en la agenda del gobierno, introdujo nuevos temas en el debate público y promovió una nueva generación de líderes con figuración y protagonismo político a nivel nacional.

El contenido de las demandas formuladas por el movimiento estudiantil, a lo largo del 2011, sobrepasó el carácter particular y sectorial que tuvo durante los años noventa y buena parte de la década siguiente (Roco, 2005; Fleet, 2011, Muñoz, 2011; Reyes y Vallejo 2013). Dicho cambio fue consecuencia de las modificaciones introducidas al sistema de financiamiento universitario, que implicó traspasar hacia los bancos la administración de los créditos, junto al debilitamiento de la educación pública básica y secundaria. Igualmente significativas fueron las protestas de estudiantes secundarios en el primer semestre del 2006, que cuestionaron por primera vez la concepción de Educación en tanto bien de consumo (Garretón, 2007:117; Navia, 2007:10; Somma, 2012:299).

Por otra parte, el movimiento del 2011 fue capaz de promover nuevos liderazgos que se proyectaron hacia otros ámbitos de la representación política. Sin embargo, en las dirigencias estudiantiles se produjeron una serie de tensiones, que inicialmente provocaron la crítica hacia el rol desempeñado por los líderes con más figuración y presencia mediática. Asimismo, se generaron disputas, diferencias y cuestionamientos entre las juventudes políticas, que no solo implicaron una crítica a su rol de voceros, sino que condicionaron la proyección del movimiento, el éxito de las movilizaciones y las negociaciones con el gobierno.

El presente artículo da cuenta de las fracturas políticas e ideológicas en las dirigencias y organizaciones estudiantiles universitarias, durante las protestas que se desencadenaron en Chile el año 2011. Se utiliza el concepto de fractura, o «clivaje», promovido por la sociología política y la ciencia política contemporánea (Lipset y Rokkan, 1967; 
Rokkan, 1999; Diani, 2000; Lipset, 2002), para diferenciar las propuestas y visiones de los dirigentes entre sí y de sus respectivas organizaciones. Además, se toman en cuenta una serie de aspectos que formaron parte de la discusión en torno a la situación de las universidades, así como las críticas hacia el sistema político en general. Al igual que en momentos anteriores a la experiencia autoritaria (Huneeus, 1973; Portantiero, 1987; Garretón y Martínez, 1985. Tomo I y Tomo IV), se asume que el movimiento estudiantil del 2011 contribuyó a la promoción de una nueva élite política y, asimismo, a la formación de nuevos referentes políticos. Como ocurrió en la segunda mitad de los años sesenta (Huneeus, 1973:VII), la proyección e impacto político del movimiento se acompañó de una tendencia al fraccionamiento y una notoria primacía del debate de carácter ideológico entre sus dirigentes.

Como hipótesis general, se afirma que las propuestas formuladas por el movimiento estudiantil, inicialmente, se instalaron sobre un nuevo «clivajes» o fractura social sustentado en la crítica a la mercantilización de la Educación Superior, y que se reconoce en la distinción Estado-Mercado. Al mismo tiempo, se instalaron sobre una crítica al funcionamiento del sistema político y el déficit democrático de la sociedad chilena, insistiendo en la necesidad de generar nuevas formas de participación y apropiación del espacio público (Fuentes, 2012; Avendaño, 2013). A estas fracturas generales se fueron agregando otras que se originaron, al calor de las movilizaciones, al interior de la dirigencia del movimiento estudiantil (Figueroa, 2012; Jackson, 2013; Reyes y Vallejo, 2013). Fracturas que se pudieron reconocer en las diferentes posturas que los dirigentes asumieron frente a la solución de los conflictos con el gobierno, la proyección del movimiento, la visión acerca de los partidos y la crítica a la democracia representativa.

\section{PROTAGONISMO POLÍTICO Y TRAYECTORIA RECIENTE DEL MOVIMIENTO ESTUDIANTIL}

\section{a) Interpretando al movimiento estudiantil y su impacto político}

Si se pone atención a los factores estructurales que desencadenaron las movilizaciones estudiantiles del 2011, es posible reconocer similitudes con lo ocurrido en algunos países de Europa occidental a fines de los años sesenta. De acuerdo a lo que sostuvo Touraine (1970), el «mayo francés» de 1968 fue consecuencia de las limitaciones que surgieron en la estructura laboral y productiva, en un contexto de aumento ex- 
ponencial de las matrículas universitarias y del número de egresados. Una tendencia similar se venía manifestando en Chile hacia los inicios de la presente década, aunque con un porcentaje de estudiantes matriculados muy superior al de la Francia de fines de los sesenta (Tironi, 2011:131). De acuerdo a lo señalado por Tironi, en ambos casos se habría evidenciado un temor entre los estudiantes a no encontrar empleos acordes a sus expectativas, con el agravante que en Chile los empleos deben servir para cubrir las deudas adquiridas durante los años de estudio. Junto a los altos costos en los aranceles, los nuevos profesionales que han ido surgiendo se han enfrentado a una verdadera segregación, según institución de origen y formación recibida, que contrasta con la sobreoferta de universidades y carreras (Waissbluth, 2011:37).

Visto desde la perspectiva de los «nuevos movimientos sociales» (Diani, 2000; Della Porta y Diani, 2006; Tarrow, 2011), el movimiento estudiantil chileno se caracterizó por establecer vínculos formales entre las organizaciones, al mismo tiempo que supo desplegar redes informales con el conjunto de los estudiantes y el resto de la comunidad, lo que explicaría su amplia y heterogénea convocatoria (Segovia y Gamboa, 2012; Urra, 2012; Varas, 2013). Por otro lado, buena parte de sus acciones fueron de tipo «contenciosas», en el sentido de Tarrow (2011), dado que por su capacidad de convocatoria logró formular demandas que constituyeron verdaderas amenazas para las autoridades y los sectores más conservadores de la sociedad chilena, especialmente por su crítica a la mercantilización y al lucro en la educación. Su éxito, en buenas cuentas, fue consecuencia de la capacidad que tuvieron sus dirigentes para aprovechar la «estructura de oportunidades» que se fue generando entre fines del 2010 e inicios del 2011, como los errores cometidos por el gobierno y la presencia de una oposición política debilitada, que no logró hacer efectivo su rol de tal.

En el momento que se configura el movimiento logra instalar una nueva fractura o «clivaje» que distingue a los partidarios de la educación pública y críticos del lucro, respecto a quienes realizan una defensa categórica del modelo educacional vigente. Esa fractura se complementó con una crítica al funcionamiento del sistema político, distinguiendo a los partidarios de una mayor profundización de la democracia de quienes se manifestaron defensores de la institucionalidad existente. ${ }^{1}$ Pero estas líneas divisorias, que pasan a ser asumidas por el

1 Por este y otros cuestionamientos anteriores, Fuentes plantea que «el nuevo 'clivaje' o línea divisoria de la elite política será entre un ideal 
conjunto de la sociedad, dan paso a otras que se generan entre los dirigentes estudiantiles y entre las organizaciones políticas que integraban el movimiento estudiantil.

Es por eso que, para efectos del análisis, se toma como referencia la teoría de los «clivajes», formulada por Lipset y Rokkan (1967) y reafirmada por ambos en años posteriores (Rokkan, 1999; Lipset, 2002). Según ellos, la existencia de ciertas fracturas en la sociedad hace posible la configuración de alineamientos sobre los cuales se erige la representación política. Los grupos políticamente organizados, tienden a situarse a un lado de la fractura para lo cual definen aspectos programáticos y tipos de acción que pretenden ser coherentes con el alineamiento asumido. Definiciones posteriores plantean ir más allá del enfoque excesivamente estructuralista, formulado por Lipset y Rokkan. De acuerdo a la lectura que hace Diani (2000) y sobre todo Deegan-Krausse y Enyedi (2010), el enfoque de Lipset y Rokkan puede resultar un tanto determinista, al considerar principalmente el peso de los factores estructurales en la definición de la fractura. Por tanto, las organizaciones políticas $-\mathrm{y}$ quienes entran en una dinámica de acción colectiva - juegan un papel central en mantener vigente una determinada fracturas, promover otras, exacerbar situaciones de conflicto existentes, o contribuir a su moderación.

Siguiendo este último planteamiento, el movimiento del 2011 promovió un nuevo «clivaje», en base al rechazo a la mercantilización de la Educación Superior, que fue incorporado en el debate público nacional. Al mismo tiempo, reafirmó su crítica al pasado autoritario, tal como el movimiento del 2006 lo había hecho con la Ley Orgánica Constitucional de Educación (LOCE), heredada del régimen militar (Garretón, 2007:115ss; Garretón, 2012:144-145). Esa crítica la reforzó con el cuestionamiento a los déficit de la democracia chilena, y sobre todo a lo que había sido la primacía del «pacto de la transición» (Fuentes, 2012; Garretón, 2012). Internamente, el movimiento experimentó otras divisiones que permiten reconocer al menos tres o cuatro sectores organizados que se disputaron la representación, y la definición del contenido de las demandas. Este mismo fraccionamiento derivó en una lucha interna por la conducción del movimiento, mediante la instrumentalización de la principal instancia de coordinación entre las federaciones estudiantiles: la Confederación de Estudiantes de Chile (CONFECH).

democrático más representativo y un ideal democrático más 'participativo'» (Fuentes, 2012:24). 


\section{b) Irrupción del movimiento estudiantil}

Entre 1992 y 1997, las organizaciones y federaciones estudiantiles de las universidades tradicionales, llevaron a cabo una serie de acciones cuyos objetivos demandaban solución al problema del financiamiento y el aumento de la ayuda crediticia de parte del Estado. Durante el año 1992, las manifestaciones que se efectuaron tanto en regiones como en Santiago demandaban la asignación del 1\% del Producto Geográfico Bruto (PGB) a la Educación Superior. ${ }^{2}$ Hubo protestas y manifestaciones que fueron más intensas en regiones que en Santiago, ya que la Universidad de Chile era la que recibía mayor aporte del Estado, y por ende mayor cantidad de becas y créditos para los estudiantes (Muñoz, 2011:120-1). Las protestas y movilizaciones más importantes de toda la década se produjeron entre mayo y junio de 1997.

Las manifestaciones de ese año se originaron como reacción a la «Ley Marco de las Universidades Estatales» propuesta por el gobierno, en la que se establecía el sistema de autofinanciamiento de dichas instituciones (De la Maza, 1999:398). Dada la baja conflictividad social y política que existía en Chile desde 1990 (Fuentes, 2012:64ss; Hidalgo, 2011:216), las manifestaciones y protestas estudiantiles que irrumpieron en el mes de mayo de 1997 destacaron por su masividad, haber logrado la paralización de la casi totalidad de los planteles durante dos meses y la cobertura que brindaron los medios de comunicación. Las demandas por financiamiento fueron complementadas con propuestas de mayor democratización y participación de la comunidad universitaria. En materia de democratización, se promovió la abolición de normativas y estructuras generadas durante el período de la intervención militar (1973-1990), que no habían sido todavía reemplazadas

2 Cabe destacar que entre 1993 y 1994 se produce un fuerte cuestionamiento a la representación políticas de las federaciones estudiantiles de las universidades de Concepción (FEC) y de Chile (FECH), hecho que llevó a la desaparición de esta última en tanto estructura organizativa, siendo reemplazada por un Consejo de Presidentes de los centros de alumnos pertenecientes a cada Facultad. Esta situación se proyectó hasta su refundación a fines de 1995, época en la que dicha organización pasa a ser conducida por dirigentes vinculados al Partido Comunista (PC), específicamente a las Juventudes Comunistas (JJCC). Los dirigentes de la FECH vinculados a las JJCC, se orientaron a la defensa de la educación pública y a la defensa del rol de la Universidad de Chile (De la Maza, 1999:398). 
y se planteó un modelo triestamental de participación y toma de decisiones.

Lo ocurrido en 1997 fue producto de acciones que asumieron principalmente las federaciones estudiantiles de las universidades pertenecientes al Consejo de Rectores de las Universidades Chilenas (CRUCH) y que recibían financiamiento de parte del Estado. En la trayectoria del movimiento estudiantil, 1997 marca un punto de inflexión y, al mismo tiempo, el inicio de una fase de organización y mayor participación. En ese período, se organiza la Confederación de Estudiantes de Chile (CONFECH), que pasó a cumplir un rol gravitante en la articulación de las luchas emprendidas por los estudiantes a nivel nacional (De la Maza, 1999:398). Además, lo ocurrido entre mayo y junio de ese año sirvió de estímulo para la organización de centros de alumnos y federaciones - hasta ese momento inexistentes - en las universidades privadas, cuya presencia era marginal dentro del movimiento estudiantil.

Aunque con menor nivel de convocatoria se desencadenaron movilizaciones estudiantiles en el mes de mayo de 1999, concentrándose principalmente en las universidades regionales de la zona norte y sur del país. Estas movilizaciones dejaron un saldo trágico, debido a la muerte de Daniel Menco, estudiante de la Universidad de Tarapacá, tras disparos efectuados por carabineros (Roco, 2005:73). En la década siguiente la CONFECH logró mantener su poder de convocatoria y su capacidad de presión frente al gobierno. Para mediados de esa década, los objetivos del movimiento estudiantil comenzaron a apuntar hacia la modificación del sistema de asignación de becas y créditos para las universidades estatales, con el fin de asegurar el ingreso de los quintiles más pobres. Luego de varias protestas y otras acciones emprendidas entre el 2004 y el 2005, además de negociaciones con el Ministerio de Educación, se logró aumentar en un 24\% los recursos para becas y créditos. Este aumento fue considerado un importante logro para las federaciones de la CONFECH - con excepción de aquellas controladas por las Juventudes Comunistas (JJCC) - que planteaban la eliminación de los créditos y su reemplazo por un sistema de arancel diferenciado (Muñoz, 2011:140).

Será precisamente la necesidad de ampliar y asegurar las becas para los estudiantes de esos quintiles de ingreso lo que detona en 2011 las primeras protestas y acciones en contra del gobierno. La primera gran acción fue la toma de la Junta Nacional de Auxilio Escolar y Becas (JUNAEB), el día 14 de abril del 2011, debido al retraso en la 
entrega de becas y pases para el uso del transporte público (Urra, 2012:25; Figueroa, 2012:54). En esta toma participaron estudiantes de la Universidad de Chile, Universidad de Santiago (USACH) y Universidad Metropolitana de Ciencias de la Educación (UMCE). Diez días antes, seis mil estudiantes de la Universidad Central — de carácter privada - decidieron paralizar sus actividades en rechazo al ingreso de la Sociedad de Inversores Norte Sur, con lo cual dicho centro de estudios dejaba de ser «sin fines de lucro» (Reyes y Vallejo, 2013:89). Lo ocurrido con los estudiantes de la Universidad Central, el retraso en la entrega de las becas y del pase escolar, así como la expectación generada por las propuestas del gobierno para la Educación Superior, generaron el ambiente que derivó en una escalada de manifestaciones y protestas en las que se vieron involucrados los estudiantes de universidades públicas y privadas.

La oportunidad para dar inicio a las movilizaciones, esperada por las juventudes políticas opositoras de izquierda y centro-izquierda, fue creada por el propio gobierno debido a varios errores comunicacionales y el tipo de decisiones adoptadas para enfrentar el conflicto. El gobierno, que había definido el 2011 como «el Año de la Educación Superior», intentó aplacar las primeras semanas de manifestaciones callejeras a través de anuncios realizados por el Presidente de la República en su Discurso del 21 de mayo. Los anuncios contemplaron el rediseño del Aporte Fiscal Indirecto, revisar la situación de los morosos del sistema de Fondo Solidario y la creación de una Subsecretaría de Educación Superior. La CONFECH manifestó su total rechazo a esos anuncios. En lo inmediato, «dieciséis universidades se declararon en toma y paro de actividades, liceos emblemáticos fueron tomados en demanda de una educación de mayor calidad, encontrándose en el mes de mayo ciento treinta y ocho colegios a nivel nacional en paro» (Varas, 2013:189). El gobierno, se vio obligado a presentar otra iniciativa, el Gran Acuerdo Nacional por la Educación (GANE), el que volvió a ser rechazo por los estudiantes.

En agosto el movimiento entra en una nueva fase, que le permitió ir superando los plazos breves con que actuó hasta fines del mes de julio. La reformulación de los plazos obedeció a dos hechos. En primer lugar, en los inicios se promovían iniciativas y acciones, desde marchas hasta la paralización de carreras y facultades, que eran evaluadas cada dos semanas. Pero llegó un momento en que la dinámica interna del movimiento asumió características de «irresistibilidad», en términos de Arendt (2004:63). Es decir, el movimiento fue adquirien- 
do fuerza propia, obedeciendo a sus mismas «leyes», sin ser controlado por persona alguna. Camila Vallejo, presidente de la FECH y principal vocera de la CONFECH en aquel entonces, reconoce abiertamente tal fenómeno: «Cuando empezamos a ver que estaba saliendo mucha gente a las calles, que con cada movilización aumentábamos en masa y en transversalidad, vimos que esto daba para otra cosa y había que replantearse este nuevo escenario. Mi primera pregunta era cómo canalizábamos esto. Sentías temor porque en un momento convocaste algo, algo que podía escaparse de las manos» (Cf. Reyes y Vallejos, 2013:96). En segundo lugar, aumentó la convocatoria a las marchas y otras manifestaciones callejeras, tanto en Santiago como en las principales ciudades de todo el país. Aumentó el número de estudiantes universitarios y al mismo tiempo se plegaron estudiantes secundarios, gremios profesionales — como el Colegio de Profesores - organizaciones sindicales, culturales y barriales (Urra, 2012:29ss).

En relación a experiencias de los noventa, un hecho inédito fue la activa participación de las federaciones de varias universidades privadas. En un comienzo, la inclusión de los estudiantes de las universidades privadas generó resistencia en algunos dirigentes de la CONFECH. Giorgio Jackson, presidente de la FEUC y, junto a Camila Vallejo, uno de los voceros de la CONFECH, recuerda: «Unos se resistían - al ingreso de las universidades privadas - porque había incertidumbre sobre la postura política de los dirigentes de dichos planteles, mientras que otros se oponían, porque iban a perder un espacio de poder, o porque pensaban erróneamente que ese espacio de la representación estudiantil nos correspondía solo a nosotros» (Jackson, 2013:73). Jackson señala que al final los dirigentes de la CONFECH que se oponían terminaron aceptando la incorporación de esos estudiantes, así como también fue aceptada la integración de la Federación Mapuche de Estudiantes.

Rápidamente, el movimiento fue ganando el respaldo de la opinión pública, dado que supo sintonizar con el malestar de la población preocupada del problema de la calidad y afectada por los altos costos de la Educación en sus diferentes niveles. Este respaldo contrastó con la evaluación negativa que el gobierno recibió de parte de la opinión pública. El 4 de agosto, día en que tuvo lugar la jornada de protesta más intensa desde 1990, la aprobación del gobierno alcanzó el veintiséis por ciento (26\%), la más baja de todo el período 2010-2014, siendo además la más baja que haya tenido alguno de los gobiernos que se sucedieron en la fase postdictatorial (CEP, 2011). Dicha jornada comenzó con un enfren- 
tamientos entre estudiantes y carabineros, debido a la negativa del gobierno de autorizar una marcha convocada por la CONFECH, culminando en la noche con protestas ciudadanas que se extendieron por los barrios de las principales comunas de Santiago (Urra, 2012:34). Para los principales dirigentes de la CONFECH, lo ocurrido en esa jornada significó el inicio de una fase, mucho más desafiante frente al gobierno, así como de proyección y diversificación de las formas de movilización: tomas, paros, huelgas de hambre, «cacerolazos», marchas, coreografías y representaciones artísticas callejeras, tanto en Santiago como en las principales ciudades de regiones (Reyes y Vallejo, 2013:102ss). Esta nueva fase, a su vez, estuvo marcada por una serie de tensiones al interior de la CONFECH, debido a las diferencias y disputas que se generaron entre las juventudes políticas que controlaban e integraban la mesa directiva de cada federación. El gobierno aprovechó esas tensiones para dilatar decisiones, manejar los tiempos y apostar al desgaste y el debilitamiento de las movilizaciones.

Las tensiones que se evidenciaron en la CONFECH no impidieron que las acciones emprendidas por el movimiento estudiantil se prolongaran de manera continua hasta inicios del 2012 (Avendaño, 2013:182ss; Segovia y Gamboa, 2012:70; Varas, 2013:192-93). Si bien las manifestaciones fueron decreciendo en los dos últimos meses, en su conjunto tuvieron una repercusión política inesperada. En primer lugar, contribuyeron a agudizar la imagen negativa hacia el gobierno, la que se había ido arraigando en la opinión pública desde enero del 2011. Al mismo tiempo, le generaron a este tensiones internas que culminaron con la renuncia, en menos de seis meses, de dos ministros de educación, Joaquín Lavín y Felipe Bulnes. En segundo lugar, redefinieron la agenda educacional del gobierno, instalaron la discusión sobre la necesidad de una reforma tributaria y, a nivel más general, de poner fin al lucro (Segovia y Gamboa, 2012:70; Varas, 2013:193). En tercer lugar, evidenciaron un grave problema desde el punto de vista de la representación política, al no existir una fuerza opositora capaz de canalizar las demandas ciudadanas. Asimismo, instalaron el debate respecto de avanzar en reformas profundas al sistema político, al ser planteada la posibilidad de plebiscitar temas de interés nacional, o de promover alternativas de cambio constitucional (Avendaño, 2013:181182). Finalmente, el tema del lucro en la educación generó controversias tanto en sectores de la oposición como del oficialismo, sacando a la luz pública el conflicto de interés que existía en algunos parlamentarios —oficialistas y de oposición-y autoridades de gobierno. 


\section{DE LA CONVERGENCIA A LA DIVERGENCIA: TENSIONES EN EL MOVIMIENTO ESTUDIANTIL}

\section{a) Los principales grupos políticos}

Desde un primer momento se aprecian, al menos, tres grupos políticos que coexisten al interior de la CONFECH y en las mesas directivas de varias federaciones. El primer grupo estuvo conformado por las Juventudes Comunistas (en adelante JJCC). Dicha organización se encuentra directamente vinculada al Partido Comunista (PC), que desde las elecciones del 2009 contaba con tres diputados. Por el hecho de que el PC era una fuerza minoritaria en el parlamento, estaba obligado a establecer vínculos con los Partidos de la Concertación. Solo de esta forma el PC podría incidir en la discusión parlamentaria y asumir de manera efectiva su rol de oposición al nuevo gobierno de derecha que había asumido en marzo del 2010. Entre el 2002 y el 2005, el PC había moderado su crítica hacia los gobiernos de la Concertación; posteriormente, entre el 2005 y el 2009 se manifestó dispuesto a apoyar a los candidatos de esa coalición en cada segunda vuelta de las elecciones presidenciales que se efectuaron en las dos ocasiones, y pocos antes para las elecciones municipales del 2008, habían negociado un pacto de omisión (Campos, Cantillana y Poveda, 2010:173ss; Riquelme y Casals, 2010).

Con el gobierno de derecha de Sebastián Piñera (2010-2014), las JJCC, que mantenían una visión crítica de los gobiernos de la Concertación, manifestaron su disposición a establecer alianzas con otras organizaciones de centro-izquierda vinculadas a esa coalición. Desde un comienzo las JJCC se entendieron muy bien con la Nueva Acción Universitaria (NAU), agrupación de la Pontificia Universidad Católica (PUC) a la que pertenecía uno de los principales voceros de la CONFECH: Giorgio Jackson. Como reconoce el propio Jackson, a inicios del 2011, con los máximos dirigentes estudiantiles pertenecientes a las JJCC existían «afinidades políticas, no solo en cuanto a la educación, sino también respecto a otros temas sociales importantes. En la FECH fue elegida Camila Vallejo, en la FEUSACH Camilo Ballesteros, Camila Donato en la UMCE, Germaín Quintana en la UTFSM sede Santiago, mientras yo era elegido en noviembre de 2010 para encabezar la federación en la UC» (Jackson, 2013:60).

El NAU se reconocía como agrupación de centro-izquierda, con dirigentes vinculados, principalmente por relaciones de parentesco, a miembros de la Concertación. Desde el año 2008, el NAU venía con- 
trolando la Federación de Estudiantes de la Pontifica Universidad Católica (FEUC). Tenía el mérito de haber desplazado al derechista Movimiento Gremial, que por muchos años mantuvo el control de la FEUC. El NAU se había propuesto influir en la discusión política nacional, especialmente en el tema educacional, evitando que quedara en manos de los actores políticos tradicionales. En el contexto de la PUC, el NAU se logró ubicar en las antípodas del gremialismo, al hacer hincapié en la participación estudiantil dentro de la Universidad: «Somos firmes defensores del rol central del estudiante universitario dentro de nuestra misma casa de estudios y en la sociedad [...]. Creemos que la universidad mejora cuando en su construcción participan los distintos miembros que la constituyen. Por este motivo es que perseguiremos la concreción de nuevas formas de participación estudiantil en los distintos ámbitos universitarios». ${ }^{3}$ A inicios del 2011, Giorgio Jackson presidió la FEUC, principal dirigente del NAU, quien junto a Camila Vallejo se convierten en los principales voceros de la CONFECH hasta septiembre de ese año.

El segundo grupo lo constituían diversas organizaciones de izquierda antisistema, calificadas por la prensa en esos años con el nombre de «ultras». Estas organizaciones participaban de la mesa directiva de la FEUSACH y controlaban importantes federaciones estudiantiles de regiones, como ocurría en la Universidad de Concepción (FEC), la Universidad de Valparaíso (FEUV) y la Universidad del BíoBío (FEUBB), entre otras (ver tabla 2). A este grupo confluían sectores de la izquierda extraparlamentaria y dirigentes identificados como anarquistas. Dentro de las organizaciones anarquistas más influyentes, sobresalía el Frente de Estudiantes Libertarios (FEL). ${ }^{4}$ Todos estos grupos consideraban que sus objetivos debían apuntar hacia transformaciones profundas y a una fuerte crítica al sistema, por sobre la lucha de tipo reivindicativa. Su modo de operar al interior de las universidades era a través de colectivos y asambleas en las cuales se privilegiaban las relaciones horizontales. Desplegaron una fuerte crítica a los partidos, incluyendo a las JJCC y rechazaron la estructura centralista de la CONFECH. Al respecto ilustrativas resultan las palabras del dirigente de la Federación Estudiantes de la Universidad de Valparaíso, Sebas-

3 Disponible en www.nau.cl.

4 El año 2011, el FEL poseía la presidencia de la Universidad del Bío-Bío. A fines de ese año conquistó la presidencia de la Universidad Arturo Prat, en Iquique, y la Universidad Austral de Valdivia. 
tián Farfán (FEUV): «Pertenezco a un movimiento de izquierda, un colectivo estudiantil que estamos en red a nivel nacional y que critica a los partidos políticos tradicionales [...] Antes se peleaba por más crédito o becas, ahora la lucha es por educación gratis». ${ }^{5}$ Estas organizaciones manifestaron un abierto rechazo a los intentos de negociación, y un cuestionamiento a la vocería de la CONFECH desempeñada por Giorgio Jackson y Camila Vallejo.

El tercer grupo estaba constituido por la llamada Izquierda Autónoma, agrupación que deriva de otra organización de izquierda, existente hasta el 2006, denominada SurDA. La SurDA tuvo una activa participación en el movimiento estudiantil de los años noventa. A fines de 1996 logró elegir a un miembro de sus filas en la mesa directiva de la FECH (Muñoz, 2011:225). Luego de una derrota sufrida en las elecciones de la FECH del año 1997, la SurDA logró conquistar la presidencia recién a fines del 2006. De manera paralela, la SurDA pudo conquistar las presidencias de otras federaciones como las de la Universidad Tecnológica Metropolitana (FEUTEM) y de la Universidad de Concepción (FEC). Dentro del ámbito universitario, la Izquierda Autónoma supo aprovechar el capital político heredado de la SurDA. Se define como izquierda antisistema, pero en el contexto de las movilizaciones del 2011 asumió una posición intermedia entre las demás agrupaciones de izquierda y del vínculo que existía entre las JJCC con sectores de centro-izquierda (ligados a la Concertación). Esta posición venía siendo adoptada desde mucho antes de dicha coyuntura: «Contribuimos desde principio de la década - entonces como SurDA y luego desde los colectivos autonomistas - a constituir y conducir el polo de la izquierda social, para contrapesar el estrategismo del izquierdismo más infantil como el entreguismo de la izquierda tradicional» (Figueroa, 2012:103). En el caso del distanciamiento con las JJCC y las juventudes de los partidos de la Concertación, la Izquierda Autónoma consideraba que a estos dos grupos: «Los unía la política de alianzas de sus respectivas orgánicas, traducida al plano social implicaba la agitación y posterior canalización del movimiento a través de la acción parlamentaria de sus partidos» (Ibid). En lo que respecta a la izquierda extraparlamentaria, existía según los autonomistas una diferencia táctica importante. Los autonomistas cuestionaban a aquellos grupos que justificaban el uso de la violencia y, al mismo tiempo, consideraban que la disputa con el adversario pasaba por una crítica

Disponible en www.lasegunda.com. 
política. «No podía levantarse - agrega Figueroa - contra la política o la institucionalidad en abstracto. Ni en genérico sin reconocer distinciones. En lugar de desordenar las líneas del sistema político, ello produciría un cierre defensivo de sus filas» (Ibid:105).

\section{TABLA 1: PRESIDENTES DE FEDERACIONES DE UNIVERSIDADES DEL CRUCH EN SANTIAGO 2005-2012}

\begin{tabular}{|c|c|c|c|c|c|}
\hline \multirow[b]{2}{*}{ AÑOS } & \multicolumn{5}{|c|}{ FEDERACIONES ESTUDIANTILES } \\
\hline & FECH & FEUC & FEUSACH & FEUTEM & FEUMCE \\
\hline 2005 & $\begin{array}{l}\text { Felipe Melo } \\
\text { Nueva } \\
\text { Izquierda }\end{array}$ & $\begin{array}{l}\text { Rodrigo de la } \\
\text { Calle DCU }\end{array}$ & $\begin{array}{l}\text { Ursula Mix } \\
\text { JS }\end{array}$ & $\begin{array}{c}\text { Sebastián } \\
\text { Valdivia } \\
(----)\end{array}$ & ---- \\
\hline 2006 & $\begin{array}{c}\text { Nicolás Grau } \\
\text { Nueva } \\
\text { Izquierda }\end{array}$ & $\begin{array}{l}\text { Claudio Castro } \\
\text { Opción } \\
\text { Independiente }\end{array}$ & $\begin{array}{l}\text { Néstor } \\
\text { Marin } \\
\text { JS }\end{array}$ & $\begin{array}{c}\text { Claudio } \\
\text { Muñoz } \\
\text { Más UTEM }\end{array}$ & ---- \\
\hline 2007 & $\begin{array}{c}\text { Giorgio } \\
\text { Boccardo } \\
\text { SurDa } \\
\end{array}$ & $\begin{array}{l}\text { Fernando Zavala } \\
\text { M. Gremialista }\end{array}$ & $\begin{array}{c}\text { Víctor } \\
\text { Rodríguez } \\
\text { JS }\end{array}$ & $\begin{array}{c}\text { Claudio } \\
\text { Muñoz } \\
\text { Más UTEM }\end{array}$ & ---- \\
\hline 2008 & $\begin{array}{c}\text { Jorge } \\
\text { Zamorano } \\
\text { U. Social }\end{array}$ & $\begin{array}{c}\text { Felipe } \\
\text { Bettancur } \\
\text { M. Gremialista }\end{array}$ & $\begin{array}{l}\text { Gonzalo } \\
\text { Ramírez } \\
\text { JS }\end{array}$ & $\begin{array}{l}\text { Pleno de } \\
\text { Presidentes }\end{array}$ & ----- \\
\hline 2009 & $\begin{array}{c}\text { Federico Hune- } \\
\text { eus } \\
\text { Nueva } \\
\text { Izquierda } \\
\end{array}$ & $\begin{array}{c}\text { Miguel Crispi } \\
\text { NAU }\end{array}$ & $\begin{array}{l}\text { Pablo } \\
\text { Moyano } \\
\text { JS }\end{array}$ & $\begin{array}{l}\text { Pleno de } \\
\text { Presidentes }\end{array}$ & ----- \\
\hline 2010 & $\begin{array}{c}\text { Julio } \\
\text { Sarmiento } \\
\text { JJCC } \\
\end{array}$ & $\begin{array}{c}\text { Joaquí Walker } \\
\text { NAU }\end{array}$ & $\begin{array}{c}\text { Camilo } \\
\text { Ballestero } \\
\text { JJCC }\end{array}$ & $\begin{array}{l}\text { Eduardo } \\
\text { Salazar } \\
\text { Atrévete }\end{array}$ & ---- \\
\hline 2011 & $\begin{array}{l}\text { Camila } \\
\text { Vallejo } \\
\text { JJCC }\end{array}$ & $\begin{array}{c}\text { Giorgio } \\
\text { Jackson } \\
\text { NAU } \\
\end{array}$ & $\begin{array}{c}\text { Camilo } \\
\text { Ballestero } \\
\text { JJCC }\end{array}$ & $\begin{array}{l}\text { David Terzán } \\
\text { U. Activa }\end{array}$ & $\begin{array}{c}\text { Camila } \\
\text { Donato } \\
\text { JJCC }\end{array}$ \\
\hline 2012 & $\begin{array}{l}\text { Eduardo Boric } \\
\text { Izq. Autónoma }\end{array}$ & $\begin{array}{l}\text { Noam } \\
\text { Titelman } \\
\text { NAU }\end{array}$ & $\begin{array}{l}\text { Sebastián } \\
\text { Donoso } \\
\text { JS }\end{array}$ & $\begin{array}{l}\text { Pleno de } \\
\text { Presidentes }\end{array}$ & $\begin{array}{c}\text { Yoxcy } \\
\text { Campos } \\
\text { Izq. Inde- } \\
\text { pendiente }\end{array}$ \\
\hline
\end{tabular}

Fuente: elaboración propia.

Comentario aparte merece la situación de las juventudes políticas de los partidos de la Concertación, que si bien participaron en las movilizaciones su protagonismo fue marginal. La Juventud Socialista (JS) que había sido muy influyente en la década de los noventa, no controlaba ninguna de las federaciones importantes. Había sido desplazada de la FECH por las JJCC a mediados de los noventa, y de presidir la FEUSACH el año 2009, al ser derrotada por el mismo sector (ver tabla 1). La derecha universitaria en este período prácticamente no figuró. Desde hacía tres años que había perdido la conducción de la FEUC, y en el momento en 
que se iniciaban las movilizaciones el 2011 la derecha controlaba solo la Federación de Estudiantes de la Universidad de Talca (FEUTAL) (Reyes y Vallejo, 2013:92). En algunos momentos, la derecha logró la presidencia de la FEC, en 1998, y luego, por primera vez en toda su historia, dirigir la FECH durante el 2004. Al perder el control de las principales federaciones, la derecha no tuvo incidencia alguna en el movimiento estudiantil, con excepción de la disputa que llevaba a cabo con el NAU dentro de la PUC.

TABLA 2: MILITANCIA DEL PRESIDENTE DE UNA MUESTRA DE SEIS FEDERACIONES REGIONALES. AL INICIO Y AL FINAL DE LAS MOVILIZACIONES 2011.

\begin{tabular}{|c|c|c|c|c|c|c|}
\hline & \multicolumn{2}{|c|}{ CONCEPCIÓN } & \multicolumn{2}{|c|}{ VALPARAÍSO } & IQUIQUE & LA SERENA \\
\cline { 2 - 7 } & FEC & FEUBB & FEUCV & FEUV & FEUAP & FEULS \\
\hline $\begin{array}{c}\text { Inicio } \\
2011\end{array}$ & $\begin{array}{c}\text { Izq. } \\
\text { Indepen- } \\
\text { diente }\end{array}$ & FEL & $\begin{array}{c}\text { Izq. } \\
\text { Autóno- } \\
\text { ma }\end{array}$ & UNE & UNE & $\begin{array}{c}\text { Izq. } \\
\text { Indepen- } \\
\text { diente }\end{array}$ \\
\hline $\begin{array}{c}\text { Final } \\
2011\end{array}$ & FUR & FEL & UNE & UNE & FEL & $\begin{array}{c}\text { Izq. } \\
\text { Indepen- } \\
\text { diente }\end{array}$ \\
\hline
\end{tabular}

Fuente: elaboración propia.

\section{b) El trabajo mancomunado en el movimiento estudiantil}

Entre las juventudes de izquierda y centro-izquierda existía un punto en común: el considerar el espacio universitario un lugar propicio para la crítica hacia la mercantilización de la educación, la segregación generada por el sistema y las consecuencias del lucro. En aquellos que controlaban las principales federaciones existía la convicción que desde ese espacio se podría «desafiar al neoliberalismo» e incluso proponer transformaciones estructurales profundas. A medida que se fueron precisando las demandas, y se hizo posible la negociación con las autoridades de gobierno, las diferencias entre ellas se agudizaron.

Un elemento esencial en el inicio del movimiento fue la continuidad política en el control de las federaciones de Santiago, por sectores como las JJCC y el NAU. ${ }^{6}$ Ello permitió mantener propuestas programá-

6 En el caso particular de la UMCE, la victoria de Camila Donato, integrante de las JJCC, significó el desplazamiento de los grupos de izquierda más radicales que controlaban hasta ese momento la federación. Co- 
ticas y, como lo decía Giorgio Jackson coincidir en una serie de temas (Jackson, 2013:60). Jackson, Vallejo y Ballesteros se convirtieron en los principales voceros de la CONFECH. De estos tres, Jackson pasó a ser una figura de consenso entre las JJCC y los autonomistas (Figueroa, 2012:107). Por otra parte, también contribuyó el contexto político de la época. El gobierno ya había enfrentado importantes protestas ciudadanas por temas ambientales y el problema del gas en Magallanes (Segovia y Gamboa, 2012:67ss; Varas, 2013:187ss). Pese a que el origen de las movilizaciones del 2011 estuvo en demandas específicas, como había ocurrido en otras ocasiones, las juventudes políticas de las federaciones esperaban el momento oportuno para enfrentar las decisiones e iniciativas que adoptara el gobierno de Sebastián Piñera, que había asumido el 11 de marzo del 2010. El hecho de que fuera la derecha la que estaba en el gobierno permitió que existiera una conducta decidida por parte de los estudiantes de izquierda y centro-izquierda, para reaccionar ante cualquier medida orientada hacia la Educación Superior.

Las JJCC y otros grupos de izquierda se prepararon desde temprano definiendo estrategias de acción y discutiendo eventuales propuestas. Según recuerda Camila Vallejo, en las JJCC «veníamos preparando el 2011 desde las vacaciones. Cachamos que había clima, primero por la llegada del Gobierno de derecha y por el anuncio en educación superior, que el 2010 ya habíamos caracterizado como agenda privatizadora» (Cf. Reyes y Vallejo, 2013:91). Durante el 2010, el Ministerio de Educación adoptó una serie de medidas destinadas a evaluar el desempeño de los establecimientos de educación primaria y secundaria, que fueron consideradas discriminadoras por sectores de la oposición y los especialistas en el área. ${ }^{7}$ Giorgio Jackson destaca el signifi-

mo recuerda Camila Donato: «Fui una presidenta refundacional, en ese tiempo no había nada. Habían asambleas súper anarcas, y cuando uno habla de anarcas no habla de anarquistas, sino que asambleas sin conducción, sin continuidad, nada [...] no tenía(n) ningún tipo de articulación» (Cf. Reyes y Vallejo, 2013:79).

7 La medida más controversial, fueron los llamados «semáforos» $\mathrm{y}$ «mapas» elaborados por el Ministro de Educación, Joaquín Lavín, destinados a evaluar y dar a conocer el desempeño de los colegios http://ciperchile.cl. Un experto en temas educacionales, Mario Waissbluth, se refirió de esta forma a las medidas adoptadas por dicho Ministerio: «Persiste la severa dispersión de resultados entre escuelas, y la segregación escolar de Chile es la segunda peor del mundo. Ricos estu- 
cado que tenía el hecho que el Presidente de la República fuera el «más rico de la historia de Chile» y que el gobierno hubiese adoptado «una agenda bien agresiva en Educación»(Jackson, 2013:58). Esa agenda habría alertado a los dirigentes estudiantiles que ya contaban con un diagnóstico respecto de la situación de la Educación chilena.

Mucho más cauta fue la reacción de la Izquierda Autónoma frente a lo que uno de sus dirigentes denomina «los primeros tropiezos del gobierno». Además de las fisuras generadas en el propio gobierno, los dirigentes autonomistas creían necesario asegurar un amplio respaldo a las movilizaciones. Según reconoce Francisco Figueroa, dirigente de esa organización y vicepresidente de la FECH en los años 2010 y 2011: «Como Izquierda Autónoma apostábamos por levantar movilizaciones a nivel nacional, pero todavía no veíamos condiciones para que eso sucediera. Por eso apostamos también a sacar al ruedo del conflicto educacional a la rectoría y al estamento académico de la Universidad de Chile. De no fructificar una movilización nacional, pensábamos, al menos podíamos abrir un proceso interno. Por fortuna, nos equivocamos» (Figueroa, 2012:55).

A pesar de las diferencias que se comenzaron a evidenciar entre los dirigentes de las juventudes políticas, existía una visión compartida respecto de la realidad chilena y sobre cuáles debían ser las transformaciones más importantes. Las palabras de Camila Vallejo así lo demuestran: «la crisis de la educación es parte de la crisis de un modelo general, neoliberal, que repercute en la educación pero que en general reproduce la desigualdad, no solamente a través de la educación sino también del sistema económico y el sistema político» (Cf. Ouviña, 2012:15). En la misma dirección, escribe Francisco Figueroa: «La vía chilena al 'desarrollo', en lugar de traer alegría y equidad como prometiera, devino pantano de vértigos ante la incertidumbre de un futuro colonizado por el dinero y de ahogo ante la arremetida de condiciones cada vez más competitivas [...] Un malestar, en definitiva, por la pérdida de control y soberanía sobre nuestro propio futuro» (Figueroa, 2012:48). Por tanto, el diagnóstico general era compartido entre los dirigentes, mientras que las diferencias se expresaban en otros planos, en especial los medios, las tácticas y el sentido de la participación.

dian con ricos, clase media con clase media, pobres con pobres». Disponible en: http://educacion2020.cl. 


\section{c) Las divergencias al interior de la CONFECH y de las federaciones}

Según Giorgio Jackson, los problemas en la CONFECH empezaron del primer día, aunque salieron a la luz pública mucho después, cuando se agudizaron los conflictos con el gobierno. Dentro de las tensiones iniciales, sobresalieron aquellas relacionadas con las decisiones y las estrategias a adoptar, como manejar los plazos, combinar marchas y acciones callejeras con la continuidad de las clases, o bien paralizar definitivamente las actividades académicas en las universidades. Pero hubo otras, de carácter ideológicas, que también se evidenciaron desde un primer momento. En el relato de Jackson: «Hubo muchas y complejas tensiones entre los bloques políticos internos de la Confech [...]. Se formaron dos bloques: los supuestamente más moderados, que incluían a los dirigentes de la Jota (JJCC), a aquellos más ligados a los partidos de la Concertación y algunos independientes; y estaban los que conformaban la llamada ultra, que eran varios grupos independientes de izquierda más extremos, algunos con teorías libertarias, otros más bien anarquistas, de todo un poco [...]. Entre esas dos coaliciones se produjeron los conflictos más serios en la Confech, que tenían que ver, casi siempre, con la estrategia o táctica que implementaríamos como organización» (Jackson, 2013:87-88).

La CONFECH poseía una estructura organizativa que, a diferencia de las federaciones que la integraban, carecía de estatutos, lo que dificulta la participación y la toma de decisiones en su interior (Reyes y Vallejo, 2013:120). Durante las movilizaciones del 2011, al interior de la CONFECH se fue imponiendo la idea de consultar a las bases sobre las decisiones adoptadas por el pleno y las acciones que se debían seguir. El hecho de estar permanentemente consultando a las bases hacía lento y dificultoso el proceso. Algunas consultas, simplemente, llegaban a niveles absurdos. Jackson relata que cuando un organismo privado invitó a los máximos dirigentes de la CONFECH a debatir sobre educación, «teníamos que preguntar a todas las federaciones de Chile si podíamos ir. Con la urgencia que existía, no podíamos demorarnos dos semanas en preparar una reunión con una ONG» (Jackson, 2013:89). En las reuniones plenarias de la CONFECH se fue perdiendo la posibilidad de alcanzar acuerdos y consensos con facilidad, como ocurría en el 2010, cuando recién se delineaban las demandas del movimiento. Macarena Godínez, que el 2010 era secretaria de comunicaciones la Federación de Estudiantes de la Universidad de Tarapacá (FEUTA) y el 2011 su presidenta, recuerda de esta forma el tenor de las 
asambleas: «El 2011 yo no fui muchas veces pero las veces que fui me encontré con un panorama muy distinto [...] en una instancia en que se iba a discutir y a resolver rápidamente los temas o a avanzar, se generaban problemas para poder discutir, porque alguien decía algo y tenía una barra atrás y la barra te aplaudía o te abucheaba, y las salas estaban muy llenas» (Cf. Reyes y Vallejo, 2013:123).

A medida que aumentaban las protestas estudiantiles y se acentuaba el conflicto con el gobierno, los grupos más radicales desarrollaron un discurso marcadamente antisistémico, rechazando toda fórmula de negociación que se realizara a través de los canales institucionales formales. Las organizaciones de este sector presidían aquellas federaciones de la CONFECH, pertenecientes a universidades regionales (ver tabla 2). ${ }^{8}$ Sin embargo, como ocurre con la FEUSACH estas organizaciones también tenían presencia en las directivas de federaciones controladas por las JJCC. Critican la postura «moderada» de estas últimas, tal como se puso de manifiesto en un rayado efectuado en la principal avenida de Santiago durante la marcha del jueves 22 de septiembre, el cual decía: «Cuidado! La Jota transa».

$\mathrm{Al}$ interior de la CONFECH, la pugna entre los grupos radicales y las JJCC fue derivando en una disputa abierta por la representación y la conducción. Hasta septiembre del 2011, la dirección de la CONFECH dependió de la función como voceros ejercida por los dirigentes de la FEUC, FECH y FEUSACH. Tras el éxito de las movilizaciones callejeas y el impacto de la jornada del 4 de agosto, Jackson Vallejo y Ballesteros aumentan su notoriedad y posicionamiento mediático, lo que les permite transformarse en figuras de opinión, influir en el debate público nacional, y llegar a ser reconocidos a nivel internacional.

El posicionamiento y la notoriedad de estos tres líderes, y sobre todo su influencia en el debate público, se tradujo en la invisibilidad de la izquierda más radical — definida por la prensa con el nombre de «ultra»- dentro del movimiento estudiantil. A fin de contrarrestar la fuerza de esos líderes y de asegurar mayor presencia en el debate público nacional, en el mes de septiembre los grupos «ultra» comienzan a plantear una serie de cuestionamientos respecto a la representación interna de la CONFECH. Se trataba de una maniobra que resultó

8 A nivel regional, solo una federación estudiantil estaba controlada por las JJCC: la Federación de Estudiantes de la Universidad de Tarapacá (FEUTA), presidida por Macarena Godinez.

9 Disponible en www.lasegunda.com. 
ser bastante efectiva para los afanes de este sector de la izquierda estudiantil. Las universidades regionales exigieron mayor presencia de sus dirigentes en la mesa central de la CONFECH, argumentando sobrerepresentación de los dirigentes de las universidades de Santiago. Eran los tres voceros, y en ocasiones Francisco Figueroa en representación de los autónomos, quienes asistían a las comisiones del Congreso y a las mesas de negociación que tuvieron que ofrecer los ministros de Educación Lavín y Bulnes.

Durante la primera quincena de septiembre del 2011, se intensificó la crítica a la vocería de Vallejo y de Jackson. Una de las razones fue la suspensión de la marcha programada para el 8 de septiembre, la otra fue la disposición manifestada por ambos líderes frente a la posibilidad de negociar con el gobierno. Esto último fue interpretado como una maniobra del propio PC, que según la «ultra» vacilaba ante las movilizaciones y apostaba por su pronta finalización. Entre las posturas críticas que surgieron al interior de la CONFECH sobresalieron las del presidente de la Federación de Estudiantes de la Universidad de Playa Ancha, quien llegó a plantear «el recambio de la vocería, en cuanto a Camila Vallejo y Giorgio Jackson, para que esta tenga postura representativa». En la misma línea la dirigencia de la Universidad Católica de Temuco planteó como moción: «Que se establezca el reemplazo de los voceros». 10

Los dirigentes estudiantiles regionales, vinculados a grupos de la izquierda radical, comenzaron a impulsar un movimiento denominado «sin FECH», a través del cual canalizaban la crítica hacia lo que consideraban el centralismo y excesivo protagonismo de los dirigentes de Santiago. De parte de la izquierda radical, el propósito de reemplazar en las vocerías a Jackson, Vallejo y Ballestero, se cumplió en el mes de septiembre cuando Patricio Contreras (Universidad de Los Lagos) y Sebastián Farfán (FEUV), fueron elegidos para asumir esa función. El cambio en la actitud de los nuevos voceros se pudo apreciar tanto en el tono amenazante de las declaraciones, así como en la crítica que recibían Jackson y los demás dirigentes de las JJCC, quienes en algunas ocasiones fueron públicamente denostados por asistentes a las asambleas de la CONFECH. Una situación de este tipo vivieron Jackson y Vallejo en una asamblea realizada por la CONFECH el día 15 de septiembre. ${ }^{11}$ La situación más extrema la vivió Giorgio Jackson, en la

10 Disponible en http://diario.latercera.com.

11 Disponible en http://diario.latercera.com. 
reunión de la CONFECH realizada el 20 de noviembre en la ciudad de Chillán, al recibir un golpe de un desconocido en el momento en que hacía una declaración pública para rechazar el uso de la violencia.

\section{LA DISPUTA IDEOLÓGICA Y PROGRAMÁTICA}

En el mes de diciembre del 2011, Guillermo Petersen, presidente de la FEC, explicaba de esta forma los conflictos que existían al interior de la CONFECH: «Hay diferencias que son como dos esquinas, dos puntas de cordones de zapatos que no se van a juntar jamás, (pero que) conviven en un espacio institucional, donde se llega a resoluciones y donde hay que limar ciertas asperezas». ${ }^{12}$ Petersen había sido parte del grupo de los «sin FECH» y en el momento de asumir la presidencia su tarea más inmediata fue reorganizar la FEC. Previo a Petersen, esta federación había sido presidida por Karol Cariola, importante dirigenta de las JJCC. Al momento de asumir la presidencia de la FEC, Petersen tendió a no acatar los acuerdos adoptados por la CONFECH, ni los llamados de los voceros de Santiago. En los primeros meses de su gestión, en vez de asumir y formar parte de las movilizaciones nacionales, la FEC privilegió efectuar acciones para resolver problemas locales.

La CONFECH y las federaciones existentes son estructuras organizativas que obligan a la elección de representantes para efectos de la deliberación. La participación horizontal y directa que se intentó fomentar a mediados de los noventa (Roco, 2005; Muñoz, 2011), fue completamente superada por la lógica de la democracia representativa. Incluso, todas las federaciones tienden a ser notoriamente «presidencialistas». Si bien los estatutos de muchas federaciones velan para que sean lo más democrática y representativas posibles, es fundamental el rol que cumple la mesa directiva central y quien ocupa la presidencia. Del mismo modo, es fundamental el rol que cumplen los voceros en la CONFECH, aunque eso implique un distanciamiento entre la estructura central y el universo de estudiantes universitarios.

Pese a las urgencias y a la intensidad de las movilizaciones, los dirigentes regionales vinculados a organizaciones más radicales insistían en fomentar la participación de las bases y la deliberación de carácter horizontal. Esta fórmula suele ser impulsada regularmente por los colectivos y agrupaciones no vinculadas a partidos, y que no aspi-

12 Disponible en www.emol.com. 
ran a serlo, en las distintas universidades del país. En el caso particular de la FEC, una vez que Petersen y su grupo denominado «Estudiantes en construcción» obtienen la presidencia, lo primero que hicieron fue promover la participación asambleísta, y la deliberación de las bases, lo que implicaba muchas veces contrariar los acuerdos y las resoluciones establecidas por la CONFECH. Esta forma de operar, en ocasiones justificaba la falta de propuestas y de una línea política coherente. Como reconocía el propio Petersen: «No teníamos programa, no teníamos nada, era el 'tollo del mono', teníamos punteos, teníamos frases nomás como: 'retornar el poder a las bases', 'construir poder estudiantil'. Un programa claro no había» (Cf. Reyes y Vallejo, 2013:85).

Pero más que la ausencia de programa, el énfasis que ciertos dirigentes ponen en la participación directa y en la existencia de estructuras centralizadas, va acompañado de un discurso antipartidista. El «antipartidismo» fue la tónica en las movilizaciones del 2011, en la totalidad de los dirigentes vinculados a la izquierda radical. Con excepción de quienes pertenecían a las JJCC, al NAU, a sectores de la Concertación y a la Izquierda Autónoma, el resto de los dirigentes formuló un discurso abiertamente antipartidista. A modo de ilustración, se puede ver la siguiente declaración de Claudio Briceño, presidente de la Federación de Estudiantes de la Universidad de Bío-Bío (FEUBB): «Me siento representado por la real izquierda que debiese existir, que no es partidista. No encierro mi horizonte de lucha en un sector que me pueda atar las manos». ${ }^{13}$ Las JJCC, el NAU y los autonomistas critican de manera categórica a los partidos tradicionales, en ese momento a los que representan la oposición dentro del Congreso Nacional, pero no reniegan de la existencia y de la función que cumplen las organizaciones partidarias. Por el contrario, la izquierda más radical, los grupos anarquistas y los colectivos niegan la existencia de los partidos y su función intermediaria.

Estos sectores de la izquierda van a formular críticas y denostaciones a las JJCC y al resto de las organizaciones vinculada a los partidos tradicionales de centro-izquierda. El antipartidismo de algunos dirigentes fue complementado con el cuestionamiento a las estructuras representativas formales, sobre las cuales se sustentaban las federaciones y la propia CONFECH, reivindicando como se ha dicho las formas horizontales de participación, a través de asambleas. Cabe destacar que esta pugna no es nueva en el movimiento estudiantil (Roco,

13 Disponible en www.lasegunda.com. 
2005:55ss). De acuerdo a los antecedentes ya presentados, la crisis que viven algunas federaciones en los años noventa - entre ellas la FECH-, lleva a promover prácticas asambleístas y a que se instale un discurso antipartidista. Una vez que esta federación se reconstruye, a mediados de los años noventa, las JJCC y demás organizaciones de centro-izquierda aceptan sin ningún reparo la lógica representativa subyacente a su modelo organizativo, combinándola con la fórmula participativa de los consejeros por facultad.

Por último, habría que señalar que el cuestionamiento hacia las JJCC y al NAU, específicamente, del que terminó sacando provecho la izquierda autónoma, obedece al intento de ciertos sectores de la izquierda de conducir el movimiento social. A nuestro juicio, se partía de una premisa errada: que controlando la CONFECH se tenía el manejo de todas las movilizaciones por la Educación. Sin embargo, como lo muestra la prensa de la época, y los análisis que se hicieron acerca de las movilizaciones, el éxito y la masividad del movimiento hasta inicios de septiembre fue producto de su heterogeneidad y diversidad. Asimismo, se trató de un movimiento que logró desarrollar dinámicas propias, donde las acciones e iniciativas espontáneas, de todos quienes se involucraron, hicieron que las expectativas que tenían los propios dirigentes fueran sobrepasadas.

\section{CONCLUSIONES}

Con las movilizaciones del 2011, la representación del movimiento estudiantil cambia de manera considerable respecto de lo que había sido en años anteriores. Vuelve a cambiar una vez que el movimiento entra en su fase cúlmine hacia fines de ese año. La disputa entre los dirigentes pasó a ser competencia política, dado que en todas las universidades se llevaron a cabo elecciones para renovar las directivas de las federaciones estudiantiles. Con excepción de algunas federaciones, entre ellas la FEUC — que se mantiene bajo la presidencia de un dirigente del NAU-, el resultado de esas elecciones significó un nuevo cambio en las dirigencias estudiantiles. Las JJCC perdieron dos de las más importantes federaciones: la FECH y la FEUSACH, además de aquella perteneciente a la UMCE. 
Existieron situaciones de continuidad en las juventudes políticas con movimientos anteriores. Hubo elementos de crítica y de carácter programático que venían siendo planteados mucho antes de los eventos del 2011. Asimismo, se dio una conexión clara con los artífices y las demandas de la movilización de estudiantes secundarios del 2006. $\mathrm{Al}$ respecto, habría que mencionar que quienes fueron estudiantes secundarios el 2006, eran estudiantes universitarios el 2011. Estas constataciones permiten hacer hincapié en la experiencia, la promoción de liderazgos, la organización y la capacidad para saber aprovechar las (estructuras de) oportunidades que ofrece el contexto político.

En su conjunto el movimiento estudiantil logró que se arraigara un nuevo «clivaje» asociado a la defensa de la educación pública, de calidad, y el cuestionamiento a su mercantilización. Como se indicó al inicio de este capítulo, también se evidenciaron otros clivajes y fracturas al interior del movimiento. Estos «clivajes» se pudieron apreciar en lo que respecta a las estructuras representativas, la función de los partidos, el modo de resolución del conflicto y el significado de la participación. Más allá de los aspectos ideológicos y políticos, el cuestionamiento que recibió el «centralismo de la CONFECH» fue algo novedoso dentro de la trayectoria del movimiento estudiantil. Pero no fue una novedad el discurso antipartidos y la valoración que se hizo de la participación y deliberación directa a través de las asambleas. Esta discusión ya se había dado en el contexto de los años noventa. Tampoco fue novedad la pugna entre las JJCC y la izquierda más radical, así como entre este último sector y las organizaciones de centro-izquierda.

Pese a las diferencias y a las disputas internas, el movimiento estudiantil del 2011 logró influir en el cambio de agenda por parte del gobierno, e incluso llegó a provocar tensiones en el propio gobierno. Adicionalmente, sacó a relucir el déficit de representación y las limitaciones que el «pacto de la transición» había permitido mantener hasta ese momento. Frente al debilitamiento de la oposición, y en especial la pasividad que tuvieron los partidos de la Concertación, el movimiento estudiantil asumió una «oposición social» y pudo desarrollar acciones de contención ante medidas que el gobierno pretendía adoptar en el ámbito de la Educación. Finalmente, los principales dirigentes estudiantiles, en especial quienes se transformaron en figuras nacionales, lograron una proyección política en eventos electorales posteriores. La experiencia de las movilizaciones del 2011 permitió consolidar y dar continuidad a agrupaciones que ya tenían un posicionamiento importante en las mesas directivas y en la conducción de 
algunas federaciones, como la Izquierda Autónoma y el NAU, así como a debilitar el protagonismo que poseían hasta entonces las JJCC.

SANTIAGO (CHILE), JULIO 2014

RECIBIDO: JULIO 2014

ACEPTADO: DICIEMBRE 2014

\section{REFERENCIAS BIBLIOGRÁFICAS}

ARENDT, HANNAH (2004): Sobre la revolución, Madrid: Alianza Editorial.

AVENDAÑo, OCTAVIO (2013): «Reformas políticas en el gobierno de Sebastián Piñera, Chile 2010-2013». Revista Mexicana de Ciencias Políticas y Sociales. Vol. 58. №218.

Campos, Javiera, Carlos Cantillana y Antonio Poveda (2010): «De vuelta al Congreso: El Partido Comunista y sus tres escaños». En M. MORALES Y P. NAVIA (eds.): El sismo electoral de 2009. Cambio y continuidad en las preferencias políticas de los chilenos. Santiago: UDP.

CENTRO DE Estudios PúBlicos (2011): «Estudio nacional de opinión pública» $N^{\circ} 6$, junio-julio. Santiago: CEP.

DeEDAN-Krause, KEVIN y Zsolt ENYEDI (2010): «Agency and the Structure of Party Competition: Alignment, Stability and the Role of Political Elites». West European Politics, Vol. 33, №3.

De la MAZA, GonZalo (1999): «Los movimientos sociales en la democratización de Chile». En P. DRAKE e I. JAKSIC (eds.): El modelo chileno. Democracia y desarrollo en los noventa. Santiago: Lom Ediciones.

Della Porta, Donatella y Mario Diani (2006): Social Movements: An Introduction, Oxford: Blackwell.

DIANI, MARIO (2000): «Simmel to Rokkan and Beyond. Towards a Network Theory of (New) Social Movements». European Journal of Social Theory. Vol. 3, №4.

FiguerOA, FRANCISCO (2012): Llegamos para quedarnos. Crónicas de la revuelta estudiantil. Santiago: Lom Ediciones.

FLEET, NICOLÁs (2011): «Movimiento estudiantil y transformaciones sociales en Chile». Polis. Vol. 10, No30.

Fuentes, Claudio (2012): El pacto. Poder, constitución y prácticas políticas en Chile (1990-2010). Santiago: UDP.

GARRETÓN, MANUEL ANTONIO (2007): Del postpinochetismo a la sociedad democrática. Globalización y política en el Bicentenario. Buenos Aires: Debate.

(2012): Neoliberalismo corregido y progresismo limitado. Los gobiernos de la Concertación en Chile, 1990-2010. Santiago: Editorial Arcis. 
— y JAVIER MARTÍNEZ (1985): Biblioteca del movimiento estudiantil. Tomo I y Tomo IV: Universidades chilenas: Historia, reforma e intervención. Santiago: Ediciones Sur.

Hidalgo, Paulo: El ciclo político de la Concertación (1990-2010). Santiago: Uqbar Editores.

HuneEus, CARLOS (1973): Movimientos universitarios y generación de élites dirigentes. Estudio de casos. Santiago: CPU.

JACKSON, GIORGIO (2013): El país que soñamos. Santiago: Debate.

LiPSET, Seymour M. (2002): «Cleavages, Parties and Democracy». En: L. KarVonen y S. KuHnle (eds.): Party Systems and Voter Alignments revisited. Londres: Routledge.

— y STEIN ROKKAN (1967): «Cleavage Structures, Party Systems, and Voter Alignments: An Introduction». En: Party Systems and Voter Aligments, Nueva York: The Free Press.

MUÑOZ T., VÍCTOR (2011): Generaciones. Juventud universitaria e izquierdas políticas en Chile y México (Universidad de Chile-UNAM, 19862006). Santiago: Lom.

NAVIA, PATRICIO (2007): «¿Qué le pasó a Bachelet?». Nueva Sociedad No212.

OUVIÑA, HERNÁN (2012): «Somos la generación que perdió el miedo. Entrevista a Camila Vallejo Dowling». Revista del Observatorio Social de América Latina. CLASCO, Año XIII, N³1.

PORTANTIERO, JUAN CARLOS (1987): Estudiantes y política en América Latina. El proceso de la Reforma Universitaria (1918-1938). México: Siglo XXI.

REYES, CAROLINA y JAVIERA VALLEJO (2013): Los días que avanzaron años. El movimiento estudiantil 2011 desde la perspectiva de sus dirigentes. Santiago: Instituto de Comunicación e Imagen, Universidad de Chile.

Roco, RoDRIGO (2005): «La FECH de fines de los 90: relatos de una historia presente». Anales de la Universidad de Chile $\mathrm{N}^{\circ} 17$.

RoKKAN, STEIN (1999): State Formation, Nation- building, and Mass Politics in Europe. Oxford: Oxford University Press.

Segovia, CARolina y Ricardo GamboA (2012): «Chile: el año en que salimos a la calle». Revista de Ciencia Política, Vol. 32, №1.

SOMMA, NiCOLÁS (2012): «The Chilean Student Movement of 2011-2012: Challenging the Marketization of Education». Interface. Vol. 4, No2.

RiQuelme, Alfredo Y Marcelo Casals (2010): «El Partido Comunista de Chile y la transición interminable». En A. VARAS, A. RIQUELME y M. CASAls (eds.). El Partido Comunista en Chile. Una historia presente. Santiago: Catalonia.

Tarrow, Sidney G. (2011): Power in Movement. Social Movements and Contentions Politics. Cambridge: Cambridge University Press.

TiRoni, Eugenio (2011): ¿Por qué no me quieren? Del Piñera way a la rebelión de los estudiantes. Santiago: Uqbar Editores.

TOURAINE, AlAin (1970): El movimiento de mayo o el comunismo utópico. Buenos Aires: Signos. 
URRA, JUAN (2012): «La movilización estudiantil chilena en 2011». Revista del Observatorio Social de América Latina. ClACSO, Año XIII, Nº31.

VARAS, AUgusto (2013): El gobierno de Piñera 2010-2014. La fronda aristocrática revivida. Santiago: Catalonia.

WAISSBLUTH, MARIO (s/f): «El semáforo 2020: Las metas de Lavín. El balance de Educación 2020 y los desafíos que vienen».

Disponible en: http://educacion2020.cl.

_ (2011): «Manifestaciones estudiantiles en Chile». Foreing Affairs Latinoamérica. Vol. 11, №4. 